\title{
Liraglutide vs insulin glargine and placebo in combination with metformin and sulfonylurea therapy in type 2 diabetes mellitus (LEAD-5 met+SU): a randomised controlled trial
}

\author{
D. Russell-Jones • A. Vaag • O. Schmitz • B. K. Sethi • N. Lalic • \\ S. Antic • M. Zdravkovic • G. M. Ravn • R. Simó • \\ on behalf of the Liraglutide Effect and Action \\ in Diabetes 5 (LEAD-5) met+SU Study Group
}

Received: 18 May 2009 / Accepted: 30 June 2009 /Published online: 14 August 2009

(C) The Author(s) 2009. This article is published with open access at Springerlink.com

\begin{abstract}
Aims/hypothesis The aim of the study was to compare the efficacy and safety of liraglutide in type 2 diabetes mellitus vs placebo and insulin glargine (A21Gly,B31Arg,B32Arg human insulin), all in combination with metformin and glimepiride.

Methods This randomised (using a telephone or web-based randomisation system), parallel-group, controlled 26 week trial of 581 patients with type 2 diabetes mellitus on prior
\end{abstract}

Electronic supplementary material The online version of this article (doi:10.1007/s00125-009-1472-y) contains a list of members of the LEAD-5 Study Group, which is available to authorised users.

D. Russell-Jones $(\bowtie)$

Department of Diabetes and Endocrinology,

Royal Guildford Hospital,

Egerton Road,

Guildford GU2 7XX, UK

e-mail: drj@royalsurrey.nhs.uk

\author{
A. Vaag \\ Department of Endocrinology, Steno Diabetes Center, \\ Gentofte, Denmark \\ O. Schmitz \\ University Hospital, University of Aarhus, \\ Aarhus, Denmark \\ B. K. Sethi \\ Care Hospitals, \\ Hyderabad, India \\ N. Lalic \\ School of Medicine, \\ Belgrade, Serbia

monotherapy $\left(\mathrm{HbA}_{1 \mathrm{c}} 7.5-10 \%\right)$ and combination therapy (7.0-10\%) was conducted in 107 centres in 17 countries. The primary endpoint was $\mathrm{HbA}_{1 \mathrm{c}}$. Patients were randomised (2:1:2) to liraglutide $1.8 \mathrm{mg}$ once daily $(n=232)$, liraglutide placebo $(n=115)$ and open-label insulin glargine $(n=234)$, all in combination with metformin ( $1 \mathrm{~g}$ twice daily) and glimepiride (4 $\mathrm{mg}$ once daily). Investigators, participants and study monitors were blinded to the treatment status of the liraglutide and placebo groups at all times.
Diabetes and Metabolic Diseases Clinic,
Niš, Serbia

M. Zdravkovic · G. M. Ravn

Novo Nordisk A/S,

Bagsvaerd, Denmark

R. Simó

CIBER de Diabetes y Enfermedades Metabólicas (CIBERDEM), Instituto de Salud Carlos III

URL: www.ciberdem/org

R. Simó

Diabetes Research Unit,

Institut de Recerca Hospital Universitari Vall d'Hebron,

Barcelona, Spain 
Results The number of patients analysed as intention to treat were: liraglutide $n=230$, placebo $n=114$, insulin glargine $n=$ 232. Liraglutide reduced $\mathrm{HbA}_{1 \mathrm{c}}$ significantly vs glargine (1.33\% vs $1.09 \% ;-0.24 \%$ difference, $95 \%$ CI $0.08,0.39 ; p=$ $0.0015)$ and placebo ( $-1.09 \%$ difference, $95 \%$ CI $0.90,1.28$; $p<0.0001)$. There was greater weight loss with liraglutide vs placebo (treatment difference $-1.39 \mathrm{~kg}, 95 \%$ CI 2.10 , $0.69 ; p=0.0001$ ), and vs glargine (treatment difference $-3.43 \mathrm{~kg}, 95 \%$ CI 4.00, 2.86; $p<0.0001)$. Liraglutide reduced systolic BP $(-4.0 \mathrm{mmHg})$ vs glargine $(+0.5 \mathrm{mmHg}$; $-4.5 \mathrm{mmHg}$ difference, 95\% CI 6.8, $-2.2 ; p=0.0001)$ but not vs placebo $(p=0.0791)$. Rates of hypoglycaemic episodes (major, minor and symptoms only, respectively) were 0.06 , 1.2 and 1.0 events/patient/year, respectively, in the liraglutide group (vs 0, 1.3, 1.8 and 0, 1.0, 0.5 with glargine and placebo, respectively). A slightly higher number of adverse events (including nausea at 14\%) were reported with liraglutide, but only $9.8 \%$ of participants in the group receiving liraglutide developed anti-liraglutide antibodies.

Conclusions/interpretation Liraglutide added to metformin and sulfonylurea produced significant improvement in glycaemic control and bodyweight compared with placebo and insulin glargine. The difference vs insulin glargine in $\mathrm{HbA}_{1 \mathrm{c}}$ was within the predefined non-inferiority margin.

Trial registration: ClinicalTrials.gov NCT00331851

Funding: The study was funded by Novo Nordisk A/S.

Keywords DPP-4 - Exenatide - Incretin · Insulin glargine · LEAD-5

$\begin{array}{ll}\text { Abbreviations } \\ \text { AACE } & \text { American Association of Clinical } \\ & \text { Endocrinologists } \\ \text { ACE } & \text { American College of Endocrinology } \\ \text { ADA } & \text { American Diabetes Association } \\ \text { AEs } & \text { Adverse events } \\ \text { ANCOVA } & \text { Analysis of covariance } \\ \text { DPP-4 } & \text { Dipeptidyl peptidase } 4 \\ \text { GLP-1 } & \text { Glucagon-like peptide-1 } \\ \text { GSRS } & \text { Gastrointestinal Symptoms Rating Scale } \\ \text { LDL-C } & \text { Low density lipoprotein-cholesterol } \\ \text { NGSP } & \text { National Glycohemoglobin Standardization } \\ & \text { Program } \\ \text { OGLA } & \text { Oral glucose-lowering drug } \\ \text { PG } & \text { Plasma glucose } \\ \text { PPG } & \text { Postprandial plasma glucose } \\ \text { TTT } & \text { Treat to target } \\ \text { VAS } & \text { Visual analogue scale }\end{array}$

\section{Introduction}

Type 2 diabetes mellitus is a progressive multi-system disease in which individuals exhibit varying degrees of declining beta cell function, insulin resistance and a failure to suppress postprandial glucagon secretion. It is associated with an array of co-morbidities and potentially devastating complications. Currently available therapies do not adequately control glycaemia in the long term as they do not address the issue of declining beta cell function and do not impact positively on weight or cardiovascular concerns associated with the disease. Furthermore, such therapies often comprise complex treatment and titration regimens and can increase the risk of hypoglycaemia and undesirable effects such as oedema and weight gain [1].

Glucagon-like peptide-1 (GLP-1) is a naturally occurring incretin hormone with a wide range of physiological actions that make it a potent blood-glucose-lowering agent with the potential to modify the natural history of type 2 diabetes [2]. In animal models, native GLP-1 stimulates beta cell proliferation, inhibits apoptosis in vitro (which may increase beta cell mass and function) and may have a number of cardiovascular and other benefits [3]. The glucose-lowering actions of GLP-1 are glucose dependent, which limits the risk of hypoglycaemia [4]. However, its very short half-life, consequent to its rapid metabolism by the enzyme dipeptidyl peptidase-4 (DPP-4), limits its therapeutic potential [2].

Liraglutide is a once-daily human GLP-1 analogue with a high degree (97\%) of amino-acid-sequence identity with native human GLP-1 [5]. The molecule has a half-life of $13 \mathrm{~h}$ making it suitable for once-daily subcutaneous administration [6-8]. Clinical data from early clinical trials demonstrate that liraglutide reduces blood glucose, bodyweight and systolic blood pressure (SBP) [9].

The objective of the present study was to compare the efficacy and safety of liraglutide with that of self-titrated basal insulin glargine (A21Gly,B31Arg,B32Arg human insulin) in patients with type 2 diabetes not adequately controlled with metformin and glimepiride.

\section{Methods}

Design overview

This 26 week randomised, parallel-group, placebo-controlled study (with liraglutide placebo and open-label insulin glargine arms) was carried out in adult patients with type 2 diabetes. 
Setting and participants

The 581 randomised patients in this multicentre (107 sites), multinational (17 countries) trial were 18-80 years old, with type 2 diabetes treated with oral glucose-lowering drugs (OGLAs) (94-95\% combination therapy) (Table 1) for at least 3 months before screening. General inclusion criteria included: $\mathrm{HbA}_{1 \mathrm{c}}$ level of $7.5-10 \%$ if on OGLA monotherapy or $7-10 \%$ if on OGLA combination therapy, and $\mathrm{BMI} \leq 45 \mathrm{~kg} / \mathrm{m}^{2}$. Patients were excluded if they: had used insulin within 3 months prior to the trial (except for short-term treatment for intercurrent illness); had impaired hepatic or renal function, clinically significant cardiovascular disease, proliferative retinopathy or maculopathy, hypertension $(\geq 180 / 100 \mathrm{mmHg}$ ) or cancer; were pregnant; experienced recurrent hypoglycaemia or hypoglycaemia unawareness; were seropositive for hepatitis $\mathrm{B}$ antigen or hepatitis $\mathrm{C}$ antibody; or used any drugs except for OGLAs that could affect blood glucose levels. The trial was conducted in accordance with the Declaration of Helsinki and ICH Good Clinical Practice. Written informed consent was obtained from patients prior to commencement of the trial, and protocols and consent forms were approved by the local health authorities, the local independent ethics committees and institutional review boards (Electronic supplementary material $[\mathrm{ESM}])$.

\section{Randomisation and interventions}

Randomisation (using a telephone or web-based randomisation system) followed a 6 week run-in period during which participants were placed on a standard combination therapy with metformin and glimepiride: forced metformin and glimepiride dose escalation over 3 weeks followed by a 3 week maintenance period. Participants already on $2 \mathrm{~g}$ metformin and sulfonylurea therapy could proceed directly to the maintenance regimen at the discretion of the investigator. During the dose-escalation period, doses of metformin and glimepiride were increased by up to $2 \mathrm{~g}$ /day and $4 \mathrm{mg} /$ day, respectively.

Patients were randomised if they met the inclusion criteria, had received glimepiride $(4 \mathrm{mg})$ and metformin $(2 \mathrm{~g})$ treatment for at least 3 weeks and had a fasting plasma glucose (FPG) between 7.5 and $12.8 \mathrm{mmol} / \mathrm{l}$ after the 6 week run-in. All patients were stratified at randomisation based on their previous therapy (OGLA monotherapy or combination therapy).

Patients were randomly allocated to three interventions (2:1:2) to receive: once-daily liraglutide (blinded); oncedaily liraglutide placebo (blinded) or once-daily insulin glargine (open-labelled; sanofi-aventis, Paris, France), all in combination with metformin and glimepiride (open-labelled). Liraglutide and liraglutide placebo were supplied by Novo Nordisk A/S (Bagsvaerd, Denmark). Investigators, participants and study monitors were blinded to the treatment status of the liraglutide and placebo groups at all times.

\section{Dosing}

The $1.8 \mathrm{mg}$ liraglutide dose selected for this study was based on previously completed phase 2 dose-range-finding studies [9]. After randomisation, patients in the liraglutide group underwent a 2 week dose escalation, starting at $0.6 \mathrm{mg}$ once daily with weekly increments of $0.6 \mathrm{mg}$, reaching a final daily dose of $1.8 \mathrm{mg}$ by the end of the second week; daily placebo injections were matched for volume. After the 2 week dose-escalation period the liraglutide dose was fixed for 24 weeks. Trial medication was administered by subcutaneous injection in the abdomen, thigh or upper arm using a pre-filled pen device (with

Table 1 Patient baseline characteristics

\begin{tabular}{|c|c|c|c|c|}
\hline Characteristic & Liraglutide & Placebo & Insulin glargine & $p$ value* \\
\hline Male/female (\%) & $57: 43$ & $49: 51$ & $60: 40$ & 0.142 \\
\hline Age (years) & $57.6(9.5)$ & $57.5(9.6)$ & $57.5(10.5)$ & 0.968 \\
\hline Duration of diabetes (years) & $9.2(5.8)$ & $9.4(6.2)$ & $9.7(6.4)$ & 0.773 \\
\hline Previously on monotherapy:combination therapy at screening (\%) & $6: 94$ & $5: 95$ & $5: 95$ & 0.802 \\
\hline Fasting plasma glucose $(\mathrm{mmol} / \mathrm{l})$ & $9.1(2.1)$ & $9.4(2.0)$ & $9.1(2.0)$ & 0.444 \\
\hline $\mathrm{HbA}_{1 \mathrm{c}}(\%)$ & $8.3(0.9)$ & $8.3(0.9)$ & $8.2(0.9)$ & 0.293 \\
\hline Body mass index $\left(\mathrm{kg} / \mathrm{m}^{2}\right)$ & $30.4(5.3)$ & $31.3(5.0)$ & $30.3(5.3)$ & 0.290 \\
\hline Weight $(\mathrm{kg})$ & $85.5(19.4)$ & $85.7(16.7)$ & $85.0(17.9)$ & 0.999 \\
\hline Systolic blood pressure (mmHg) & $135(15.0)$ & $133(14.0)$ & $133(14.7)$ & 0.244 \\
\hline Diastolic blood pressure $(\mathrm{mmHg})$ & $80.8(9.1)$ & $80.4(9.3)$ & $80.5(8.0)$ & 0.962 \\
\hline
\end{tabular}

Values are mean (SD)

${ }^{*} p>0.05$ indicates no significant difference between the three treatments 
a NovoFine $30 \mathrm{G}$ disposable needle; Novo Nordisk). The injection could be administered at any time of the day. Participants were encouraged to inject liraglutide during the same overall time period on a day-to-day basis.

Reduction of glimepiride dose from $4 \mathrm{mg}$ to $2 \mathrm{mg}$ was allowed after randomisation if necessitated by adverse events (AEs) or hypoglycaemia.

As in similar previous studies [10, 11], insulin glargine (100 IU/ml injected once daily with OptiSet; sanofi-aventis) was titrated by patients following instruction by the investigator according to a specific and widely adopted dosing algorithm for insulin glargine based on fasting concentration of blood glucose (adapted from A Trial comparing Lantus Algorithms to achieve Normal blood glucose Targets in patients with Uncontrolled blood Sugar [AT-LANTUS]) [11]. The starting dose of insulin glargine was numerically equivalent to the highest $\mathrm{FPG}$ value in $\mathrm{mmol} / \mathrm{l}$ over the previous 7 days (e.g. if the FPG measure was $10 \mathrm{mmol} / \mathrm{l}$, the initial glargine dose would be $10 \mathrm{IU}$ ). This facilitated ease of initiation in this patient-driven titration. During the first 8 weeks of treatment, the dose was titrated twice weekly by the participant, based on self-measured FPG, aiming for a target value of $\mathrm{FPG} \leq 5.5 \mathrm{mmol} / \mathrm{l}$. After 8 weeks of treatment, the frequency of monitoring and titration was at the investigator's discretion, but at minimum the insulin glargine dose was adjusted at the 12 and 18 week visits. The investigator reviewed the doses and these could be changed at his/her discretion. The injection could be administered at any time of the day, but the selected time of the day remained the same throughout the trial.

Treatment allocation

Participants eligible for randomisation were assigned to liraglutide, liraglutide placebo or insulin glargine in a 2:1:2 randomisation pattern. At screening, all participants were given a 6 digit participant number. At randomisation, eligible participants were allocated to one of the three treatment groups using a telephone- or web-based randomisation system. Participants were stratified by whether they were treated with monotherapy or combination therapy for their diabetes at screening. A block size of five was defined for both groups. The blinded electronic-sealed codes were accessible to the investigators, affiliate and international product safety personnel or any other relevant party who might have had a need for breaking the treatment code; for example, for safety or regulatory purposes. Break of the code was performed through the telephone- or web-based randomisation system via an emergency code-break call. Novo Nordisk trial personnel, international product safety personnel, and the investigator received a notification generated by the interactive voice response system/ interactive web response system via an emergency code-break call.

\section{Outcomes and follow-up}

The primary efficacy outcome measure was change in whole blood $\mathrm{HbA}_{1 \mathrm{c}}$ after 26 weeks of treatment. The secondary outcome measures included changes in bodyweight, waist circumference, FPG, eight point plasma glucose (PG) profiles, beta cell function (proinsulin to $\mathrm{C}$-peptide ratio) and $\mathrm{BP}$.

$\mathrm{HbA}_{1 \mathrm{c}}$ was measured (at baseline and at weeks 12, 18 and 26) using high-performance liquid chromatography according to an assay certified by the National Glycohemoglobin Standardization Program (NGSP). Self-measurement of PG from capillary samples was performed using a Medisense Precision Xtra/MediSense Optimum glucose meter (Abbott, Maidenhead, UK) that used test strips calibrated to plasma values. Insulin and C-peptide were measured in serum using a chemiluminescence immunoassay. Proinsulin was measured in serum using an enzyme-linked immunosorbent assay. Glucagon was measured by RIA in aprotinin-treated plasma.

Safety assessments included hypoglycaemic episodes based on symptoms and PG $(<3.1 \mathrm{mmol} / \mathrm{l})$. Episodes requiring third-party medical assistance were classified as major. Liraglutide antibodies were measured by radioprecipitation assay using ${ }^{125}$ I-labelled liraglutide tracer and polyethylene glycol precipitation. Vital signs, ECG, calcitonin and biochemical and haematology measures were recorded. All AEs, either observed by the investigator or reported spontaneously by the participants, were recorded by the investigator. The participants were asked at each post-screening visit or contact with the site if they had had any AEs (including changes in concomitant illness or new illnesses) since the last evaluation. The participant was asked: 'Have you experienced any problems since the last contact?'

The trial comprised a 2 week screening period followed by a 3 week dose-escalation period, a 3 week maintenance period and then a 26 week treatment period. The follow-up period was 1 week. In total the participants attended nine visits at the site and had two telephone contacts (week 1 and 4 post-randomisation) during the trial duration of a maximum of 36 weeks. At screening, the participants were assigned a unique participant number. Inclusion and exclusion criteria were reviewed prior to randomisation. The randomisation criteria were reviewed at the randomisation visit. At each visit, information was collected and/or procedures performed according to the protocol. Patients recorded information in diaries between the visits to be reviewed at each visit. Week 26 
was the end-of-trial visit for completers, who also attended a follow-up visit (week 27). Participants with a confirmed FPG reading $>13.3 \mathrm{mmol} / \mathrm{l}$ at week 8 or thereafter and no intercurrent treatable illness were withdrawn from the study.

\section{Statistical analyses}

The data were analysed for the intent-to-treat population, defined as patients who were exposed to at least one dose of trial product(s) after randomisation. For the primary endpoint, $\mathrm{HbA}_{1 \mathrm{c}}$, the statistical analysis was also performed without the last observation carried forward on the perprotocol population (participants completing the study without significant protocol violations) (ESM Table 1). Each endpoint was analysed using an analysis of covariance (ANCOVA) model with treatment, pre-treatment and country as fixed effects and baseline as the covariate. Missing baseline values were not imputed; that is, participants without a baseline value were excluded from the primary analysis. Post-baseline missing values were replaced using last observation carried forward. Sample size calculations were based on predicted $\mathrm{HbA}_{1 \mathrm{c}}$ and bodyweight after 26 weeks of treatment.

The study was powered against the primary endpoint change in $\mathrm{HbA}_{1 \mathrm{c}}$. The non-inferiority margin against glargine was set to $0.4 \%$ and the difference to detect superiority against placebo was set to $0.5 \%$. In addition the study was powered to detect a $3 \%$ difference in weight. The combined power was greater than $85 \%$.

For superiority and non-inferiority of liraglutide vs comparators, hierarchical tests were conducted. The primary endpoint was the change from baseline in $\mathrm{HbA}_{1 \mathrm{c}}$ after 26 weeks of treatment. A sequential testing procedure was employed to protect the overall type 1 error rate. First, superiority of liraglutide to that of placebo had to be declared, then non-inferiority against glargine was tested and, if declared, superiority was tested. Finally, a test for superiority of insulin glargine vs placebo was performed.

The proportion of participants achieving $\mathrm{HbA}_{1 \mathrm{c}}$ targets (American Diabetes Association [ADA] target: <7\% [12]; American Association of Clinical Endocrinologists [AACE] target $\leq 6.5 \%$ [13]) was compared between treatments using a logistic regression model with treatment as fixed effect and baseline $\mathrm{HbA}_{1 \mathrm{c}}$ as a covariate. Hypoglycaemic episodes were analysed using a generalised linear model including treatment and country as fixed effects. Other safety data were compared by descriptive statistics. Values are expressed as means \pm SD unless otherwise noted; significance was set at $p<0.05$. For the primary analysis (non-inferiority/superiority) the level of significance was $2.5 \%$ (one-sided test) and 5\% (two-sided test).

\section{Results}

Demographics and disposition

A total of 973 patients were screened, 581 were randomised and 522 completed the study (Fig. 1). The highest withdrawal rate was seen in the placebo group, which was mainly driven by withdrawal because of ineffective therapy at a level of $11.3 \%$. AE withdrawals occurred for $4.7 \%$ (liraglutide), $0.9 \%$ (placebo) and 2.1\% (insulin glargine) of participants. Treatment groups were well matched (Table 1). The average dose of insulin glargine was $24 \mathrm{IU} /$ day at the end-of-trial visit, giving $20 \%$ and $40 \%$ of the group reaching an $\mathrm{FPG} \leq 5.5$ and $<6.7 \mathrm{mmol} / \mathrm{l}$, respectively. The average daily doses of glimepiride at the end of the study were $3.4 \mathrm{mg}$ in the liraglutide group, $3.9 \mathrm{mg}$ in the placebo group and $3.6 \mathrm{mg}$ in the insulin glargine group.

\section{Efficacy}

$\mathrm{HbA}_{1 c}$ After 26 weeks of treatment, the $\mathrm{HbA}_{1 \mathrm{c}}$ reduction from baseline with liraglutide was $1.33 \%$ (SEM 0.09) (final mean $\mathrm{HbA}_{1 \mathrm{c}}$ 7.0\%), with placebo $0.24 \%$ (SEM 0.11) (final mean $\left.\mathrm{HbA}_{1 \mathrm{c}} 8.1 \%\right)$ and with insulin glargine $1.09 \%(0.09$ SEM) (final mean $\mathrm{HbA}_{1 \mathrm{c}}$ 7.2\%) (Fig. 2). The lowest $\mathrm{HbA}_{1 \mathrm{c}}$ level was observed at 12 and 18 weeks for liraglutide and insulin glargine, respectively. Thereafter both groups had a minor increase towards week 26. The reduction in $\mathrm{HbA}_{1 \mathrm{c}}$ with liraglutide was significantly greater than the reductions observed in both the placebo and insulin glargine groups (treatment differences for liraglutide vs placebo $-1.09 \%$, 95\% CI $-1.28,-0.90 ; p<0.0001$ and liraglutide vs insulin glargine $-0.24 \%, 95 \% \mathrm{CI}-0.39,-0.08 ; p=0.0015)$. The $\mathrm{HbA}_{1 \mathrm{c}}$ reduction in the insulin glargine group was also significantly greater than in the placebo group (treatment difference $-0.85 \%, 95 \%$ CI $-1.04,-0.66 ; p<0.0001)$. Similar results for $\mathrm{HbA}_{1 \mathrm{c}}$ were achieved using the perprotocol analysis population (data not shown).

Significantly more patients reached $\mathrm{HbA}_{1 \mathrm{c}} \mathrm{AACE}$ targets of $\leq 6.5 \%$ [13] and ADA targets of $<7 \%$ [12] with liraglutide than with either placebo or insulin glargine (ESM Fig. 1).

Bodyweight The mean weight loss from baseline of $1.8 \mathrm{~kg}$ (SEM 0.33) achieved in the liraglutide group was significantly superior to the reduction in the placebo group (0.42 kg [SEM 0.39]; weight difference of $-1.39 \mathrm{~kg}, 95 \%$ CI $-2.10,-0.69 ; p=0.0001$ ) (Fig. 3). Weight increased by $1.6 \mathrm{~kg}$ (SEM 0.33) with insulin glargine, resulting in a mean treatment difference of $-3.43 \mathrm{~kg}(95 \%$ CI -4.00 , $-2.86 ; p<0.0001$ ) (Fig. 3). In addition, the reduction in waist circumference for the liraglutide group $(1.50 \mathrm{~cm})$ was 


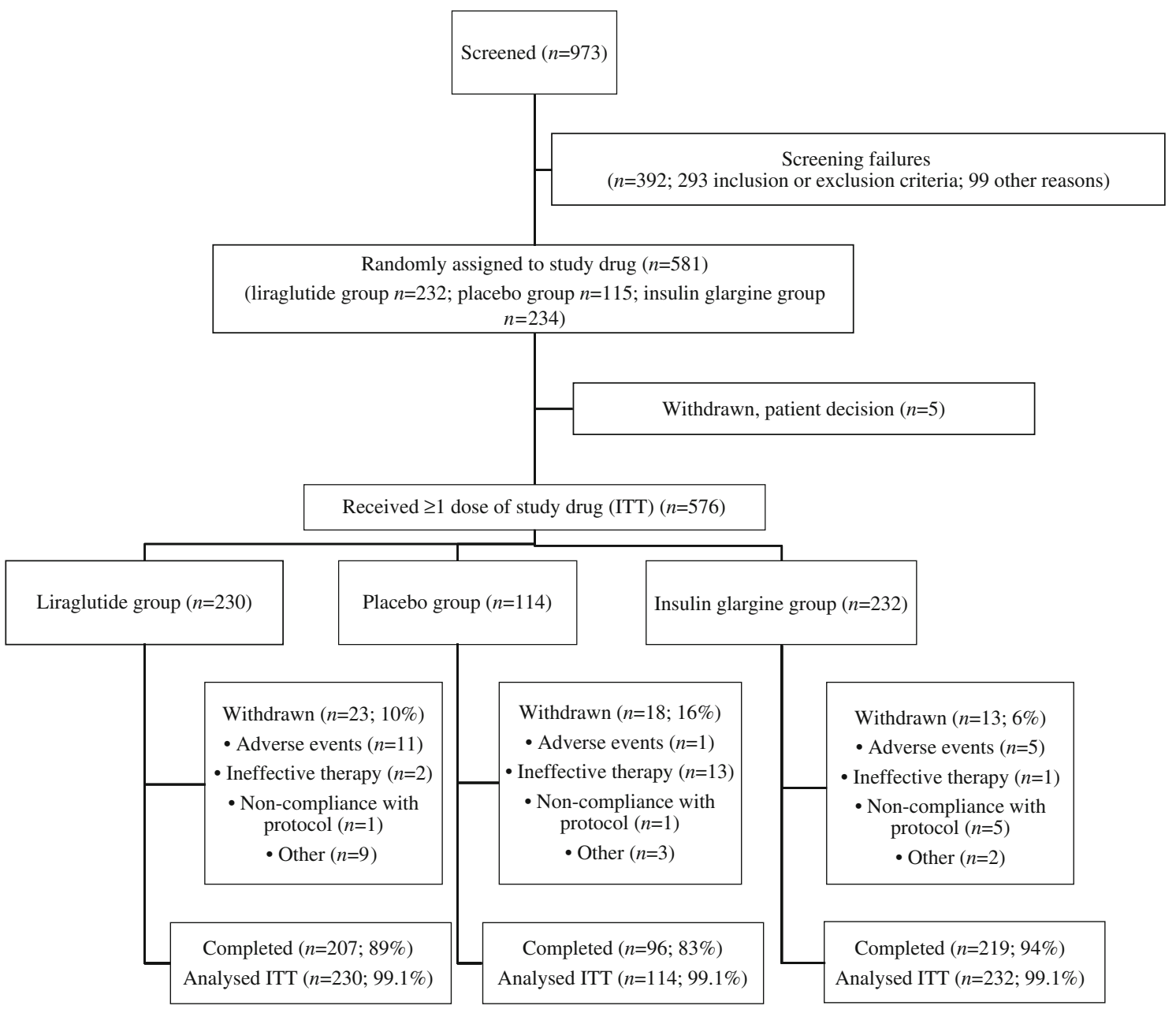

Fig. 1 Flow of patients through the study

statistically significant compared with the insulin glargine group $(0.89 \mathrm{~cm}$ increase; treatment difference $-2.40 \mathrm{~cm}$, $95 \%$ CI $-3.14,-1.65 ; p<0.0001)$, but not compared with the placebo group $(0.62 \mathrm{~cm}$ reduction; treatment difference $-0.88 \mathrm{~cm}, 95 \% \mathrm{CI}-1.81,0.04 ; p=0.0608)$.

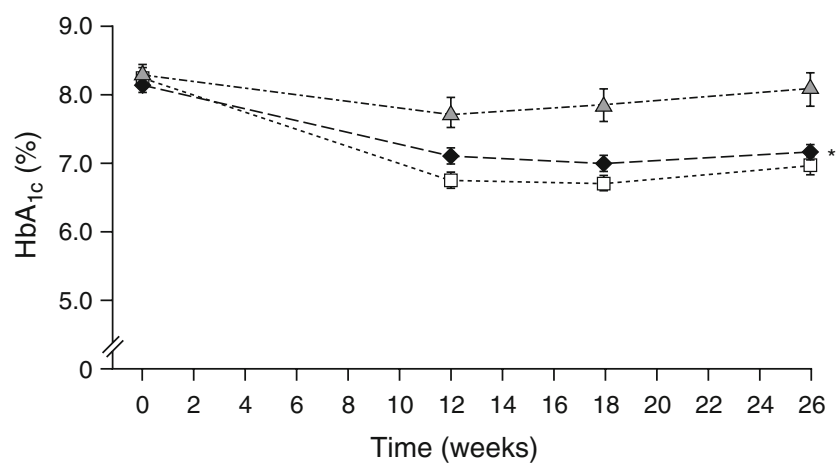

Fig. $2 \mathrm{HbA}_{1 \mathrm{c}}(\%)$ over time (last observation carried forward, intention-to-treat population). Liraglutide $1.8 \mathrm{mg}$ (squares); glargine (diamonds); placebo (triangles); ${ }^{*} p<0.05$ vs glargine and placebo
Overall, weight loss was independent of nausea, although in the very small number of patients $(n=8)$ with sustained nausea, there seemed to be a greater weight loss $(-3.2 \mathrm{~kg})$.

FPG FPG decreased rapidly with liraglutide (ESM Fig. 2); final reductions in FPG from baseline were $1.55 \mathrm{mmol} / \mathrm{l}$ for liraglutide and $1.79 \mathrm{mmol} / \mathrm{l}$ for insulin glargine, with an increase of $0.53 \mathrm{mmol} / 1$ for placebo. The reduction in mean FPG in the liraglutide group, and the likelihood of achieving ADA targets (FPG 5-7.2 mmol/l) was significantly superior compared with the placebo group (treatment difference for FPG $-2.08 \mathrm{mmol} / 1$ [95\% CI 2.53, -1.64], $p<$ 0.0001 and odds ratio $4.99,95 \%$ CI $2.65-9.39$ ) but not vs the insulin glargine group.

Postprandial plasma glucose A reduction in postprandial plasma glucose (PPG) from baseline was observed in the liraglutide $(1.81 \mathrm{mmol} / \mathrm{l})$ and insulin glargine $(1.61 \mathrm{mmol} / \mathrm{l})$ groups, with the reduction with liraglutide being significantly greater than with placebo $(0.03 \mathrm{mmol} / \mathrm{l}$; treatment difference $-1.84 \mathrm{mmol} / 1,95 \% \mathrm{CI}-2.36,-1.33 ; p<0.0001)$ but not vs 

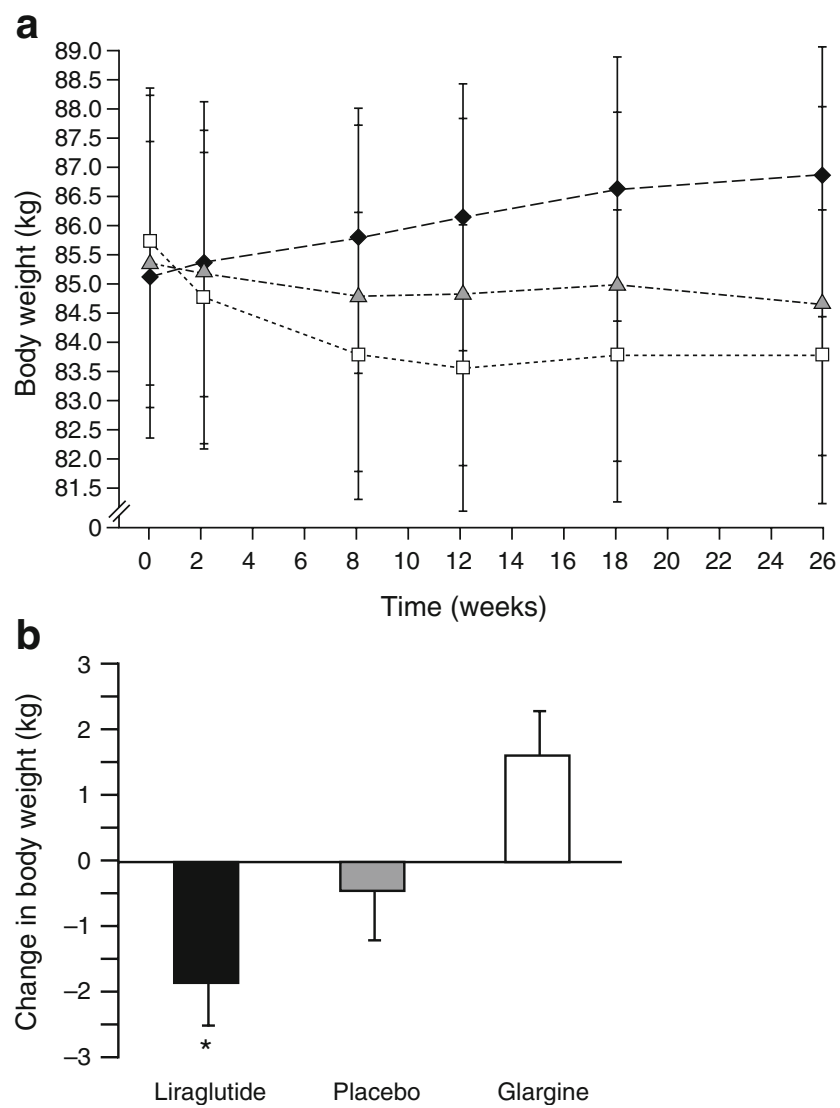

Fig. 3 a Change in bodyweight over time (mean) (last observation carried forward, intention-to-treat population). Liraglutide $1.8 \mathrm{mg}$ (squares); glargine (diamonds); placebo (triangles). b Change in bodyweight from baseline (mean [SD]) (last observation carried forward, intention-to-treat population). ${ }^{*}$ Significant vs glargine $(p<$ $0.0001)$ and placebo $(p=0.0001)$

insulin glargine. There was a statistically significantly higher likelihood of achieving ADA targets for PPG $(\leq 10 \mathrm{mmol} / \mathrm{l})$ $(p<0.0001)$ with liraglutide vs placebo.

Beta cell function The proinsulin to C-peptide ratio showed a significant improvement in the liraglutide group compared with the insulin glargine group (treatment difference $-0.00366,95 \% \mathrm{CI}-0.00597,-0.00136 ; p=0.0019)$ and the placebo group (treatment difference $-0.00671,95 \%$ CI $-0.00964,-0.00377 ; p<0.0001)$.

Cardiovascular effects A significant reduction in SBP was observed with liraglutide (4.0 $\mathrm{mmHg}$ reduction) compared with insulin glargine $(0.54 \mathrm{mmHg}$ increase; treatment difference $-4.51 \mathrm{mmHg}, 95 \% \mathrm{CI}-6.82,-2.20 ; p=0.0001$ ), but not in comparison with placebo $(1.4 \mathrm{mmHg}$ reduction; treatment difference $-2.53 \mathrm{mmHg}, 95 \% \mathrm{CI}-5.36,0.29$; $p=0.0791$ ). No significant difference in the reduction in diastolic BP was observed relative to either comparator. Based on the time course of SBP and weight reductions, the reduction in SBP occurred before substantial weight loss.
The pulse rate increased by an estimated mean 2.62 beats/ $\mathrm{min}$ in the liraglutide group, 0.08 beats/min in the glargine group (treatment difference 2.54 beats $/ \mathrm{min} ; 95 \%$ CI 1.10, 3.98; $p=0.0006$ ) and 0.93 beats $/ \mathrm{min}$ in the placebo group (treatment difference 1.70 beats $/ \mathrm{min} ; 95 \% \mathrm{CI}-0.06,3.45 ; p=0.0582$ ).

\section{Safety}

Hypoglycaemic episodes The proportion of patients experiencing minor hypoglycaemia ( $\mathrm{FPG}<3.1 \mathrm{mmol} / \mathrm{l}$ ) during the treatment period in the liraglutide group (27.4\% patients) was not different from the insulin glargine group (28.9\%) but higher compared with the placebo group (16.7\%). The rate of hypoglycaemic episodes (major, minor and symptoms only, respectively) was $0.06,1.2$ and 1.0 events/patient/year in the liraglutide group; $0,1.3$ and 1.8 events/patient/year in the glargine group; and $0,1.0$ and 0.5 events/patient/year in the placebo group.

Five patients reported major hypoglycaemic events in the liraglutide group $(2.2 \%$; only one required medical assistance and none resulted in coma or seizures). None of these was nocturnal. No major events were reported in the glargine group or in the placebo group.

Adverse events The most common AEs in the liraglutide group were gastrointestinal and of mild or moderate severity (mainly nausea) (Table 2). Altogether, four patients withdrew within 2 weeks with a gastrointestinal AE. Nausea occurred in $14 \%$ of patients in the liraglutide group, decreasing after 1-3 weeks of treatment and reaching a stable level (1.5\%) after 14 weeks; diarrhoea occurred in $10 \%$ of patients and vomiting in $7 \%$.

Although AEs were reported more frequently for the liraglutide group, serious AEs were reported less frequently in the liraglutide group (4\%) than in the placebo (7\%) and insulin glargine groups $(7 \%)$. No case of pancreatitis was reported. A summary of AEs has been provided as ESM Table 2.

After 26 weeks there was a comparable and significant increase in calcitonin levels for both liraglutide and insulin glargine vs placebo. The estimated mean calcitonin level at 26 weeks was within the normal range for participants treated with both liraglutide and insulin glargine. Antibodies to liraglutide were present in $9.8 \%$ of patients in the liraglutide group. The antibodies did not appear to alter the glucose-lowering effect of liraglutide.

\section{Discussion}

In this study, we found that liraglutide, administered once daily, was statistically superior to placebo in combination 
Table 2 Individual adverse events with frequency $>5 \%$

${ }^{*} p>0.05$ indicates no significant difference between the three treatments

$E$, number of events; $n$, number of patients experiencing the $\mathrm{AE}$; $\%$, percentage experiencing adverse event

\begin{tabular}{|c|c|c|c|c|c|c|c|c|c|c|}
\hline \multirow[t]{2}{*}{ Adverse event } & \multicolumn{3}{|c|}{ Liraglutide } & \multicolumn{3}{|c|}{ Placebo } & \multicolumn{3}{|c|}{ Insulin glargine } & \multirow[t]{2}{*}{$p$ value* } \\
\hline & $n$ & $\%$ & $E$ & $n$ & $\%$ & $E$ & $n$ & $\%$ & $E$ & \\
\hline Nausea & 32 & 13.9 & 41 & 4 & 3.5 & 4 & 3 & 1.3 & 3 & $<0.0001$ \\
\hline Diarrhoea & 23 & 10.0 & 29 & 6 & 5.3 & 7 & 3 & 1.3 & 6 & $<0.0001$ \\
\hline Dyspepsia & 15 & 6.5 & 16 & 1 & 0.9 & 1 & 4 & 1.7 & 4 & 0.0042 \\
\hline Vomiting & 15 & 6.5 & 18 & 4 & 3.5 & 4 & 1 & 0.4 & 1 & 0.0005 \\
\hline Nasopharyngitis & 21 & 9.1 & 23 & 10 & 8.8 & 10 & 26 & 11.2 & 32 & 0.6864 \\
\hline Headache & 22 & 9.6 & 33 & 9 & 7.9 & 14 & 13 & 5.6 & 18 & 0.2687 \\
\hline
\end{tabular}

with metformin and glimepiride in patients whose diabetes was inadequately controlled on these OGLAs. And while a statistically significant difference vs insulin glargine was also observed, this is of uncertain clinical relevance. Patients were more likely to meet ADA and AACE $\mathrm{HbA}_{1 \mathrm{c}}$ targets with liraglutide compared with insulin glargine. In addition, beta cell function also improved with liraglutide. Major hypoglycaemic events were observed in the liraglutide group, and not in the placebo and insulin glargine arms. The present study is the first study comparing a $24 \mathrm{~h}$-acting GLP-1 analogue with the basal insulin glargine and therefore provides information as to treatment choices in patients requiring further intensification of therapy following treatment with metformin and a sulfonylurea.

The $\mathrm{HbA}_{1 \mathrm{c}}$ reduction from baseline with liraglutide in the present study was in line with that reported by Vilsbøll and colleagues in a smaller phase 2 study, where reductions of 1.45 percentage points were achieved from similar baseline $\mathrm{HbA}_{1 \mathrm{c}}$ levels of $8.1-8.5 \%$ [9]. In a study similar to the ours, Heine and co-workers compared the exendinbased GLP-1 agonist exenatide and insulin glargine but found no difference in change in $\mathrm{HbA}_{1 \mathrm{c}}$ between the two agents $\left(\mathrm{HbA}_{1 \mathrm{c}}\right.$ reduction of $1.11 \%$ with both agents) [10].

Insulin glargine in this study lowered $\mathrm{HbA}_{1 \mathrm{c}}$ by $1.09 \%$ vs baseline. This reduction in $\mathrm{HbA}_{1 \mathrm{c}}$, and the dose of insulin used, was comparable with other insulin glargine titration trials where titration is driven by patients, and not via intense patient-carer contact schedules [10, 14-17]. Studies such as AT-LANTUS [11] and Glycemic Optimization with Algorithms and Labs at Point of Care (GOAL A1c) [18] show that patients can be encouraged to use higher insulin doses with slightly higher end-of-study $\mathrm{HbA}_{1 \mathrm{c}}$ levels than were achieved in the present study (although baseline $\mathrm{HbA}_{1 \mathrm{c}}$ levels were higher). Where more intensive titration is undertaken and insulin doses have been pushed higher, studies have achieved somewhat greater reductions in $\mathrm{HbA}_{1 \mathrm{c}}$ at the expense of concomitantly higher levels of hypoglycaemia (and weight gain) [14-21]. Nevertheless, the insulin glargine doses in this study are valid as they were consistent with documented real-life scenarios and so the data shown actually represent the most likely clinical expectations. It would, however, be interesting to compare liraglutide and glargine using a treat-totarget approach in future studies, perhaps standardising non-pharmacological interventions such as lifestyle and patient education.

We found no significant difference between fasting and postprandial control between liraglutide and insulin glargine. The postprandial data are in contrast to data from a recently conducted study where a flattening of the postprandial curve in the $5 \mathrm{~h}$ period after a standardised meal was observed [22]. In addition to its effect on glycaemic control, liraglutide treatment resulted in significant weight loss compared with placebo $(-1.4 \mathrm{~kg}$ difference) and a favourable weight difference compared with insulin glargine $(-3.4 \mathrm{~kg})$. This is consistent with previous liraglutide studies [9] where the weight loss vs placebo was in a similar range.

As with previous liraglutide studies [23], a reduction in SBP was observed vs insulin glargine, but not in comparison with placebo. Based on the time course of SBP and weight reductions, the reduction in SBP occurred before substantial weight loss occurred. The mechanism underlying the reduction in SBP observed in patients with type 2 diabetes treated with liraglutide is unknown, but it has been demonstrated that infusion of native GLP-1 increases natriuresis in humans [24]. The long-term safety of liraglutide and its impact on micro- and cardiovascular outcomes are yet to be established (as is also the case for metformin and sulfonylurea).

Although gastrointestinal AEs were reported more frequently for the liraglutide group, they were mainly mild to moderate and transient. This is in line with a study where patient self-assessments of gastrointestinal side effects with liraglutide were mainly reported in the first weeks after initiation [25]. In particular, the withdrawal rate in the present study was low and only very few cases included gastrointestinal side effects. In addition, the superior efficacy and positive impact of liraglutide on weight and weight concern have been associated with clinically important improvements in physical and emotional domains of quality of life regardless of AEs [26]. 
Overall rates of hypoglycaemia were higher in the liraglutide group as compared with the placebo group, but not different from the insulin glargine group. Five participants treated with liraglutide reported six major hypoglycaemic events. The combination of a sulfonylurea with a GLP-1 analogue increases the risk of hypoglycaemia, as has also been reported with exenatide [10, 27, 28]. As also observed in the present study, an increased risk of major hypoglycaemic events has been seen in studies where liraglutide has been combined with a sulfonylurea [29]. The mechanism behind this may reflect an uncoupling of the glucose-dependent insulin secretion of GLP-1 when combined with a sulfonylurea [30]. A low frequency of participants with liraglutide antibodies was observed; these antibodies did not appear to reduce the efficacy of liraglutide.

There are certain limitations of this trial that need to be considered. These include the open-label design of the insulin glargine arm, the relatively short duration of treatment assessed and the need for titration of insulin glargine dose in the third study arm (although proven titration algorithms were followed). Furthermore, the study participants had not previously been treated with insulin.

In conclusion, this 26 week trial demonstrated that the once-daily human GLP-1 analogue liraglutide added to combination therapy with metformin and glimepiride in patients with type 2 diabetes resulted in statistically significant superior glycaemic control compared with insulin glargine, but the difference was within the predefined non-inferiority margin of 0.4 percentage points. Liraglutide resulted in weight loss, in contrast with placebo and with insulin glargine, which caused weight gain. Major hypoglycaemic events were reported in five participants treated with liraglutide.

Acknowledgements The study was funded by Novo Nordisk A/S. All authors have been involved at each draft stage, and have seen and approved the final draft for submission. In collaboration with the investigators, Novo Nordisk was responsible for the study design, protocol, statistical analysis plans, oversight, analysis and reporting of the results. Data were recorded at the clinical centres and maintained by the sponsor. The LEAD-5 met+SU study group had full access to the data. Final responsibility for the decision to submit the manuscript for publication was with the authors, including those from Novo Nordisk. The authors gratefully acknowledge the assistance of the LEAD-5 met+SU-study group, their staff, clinical trial personnel, and the participants for participating in the study. We thank J. Beeso of Watermeadow Medical plc for medical writing services, which were financially supported by Novo Nordisk.

Duality of interest statement The authors have the following potential competing interests to declare. D. Russell-Jones: research support, Novo Nordisk. A. Vaag: member of Novo Nordisk advisory panel; consultant to Novo Nordisk; employee of Novo Nordisk; research support, Novo Nordisk. O. Schmitz: member of Amylin Pharmaceuticals advisory panel. B. K. Sethi: none reported. N. Lalic: none reported. S. Antic: none reported. M. Zdravkovic: employee of Novo Nordisk; spouse,
Speakers Bureau and stock/shareholder, Novo Nordisk. G. M. Ravn: employee of Novo Nordisk. R. Simó: none reported.

Open Access This article is distributed under the terms of the Creative Commons Attribution Noncommercial License which permits any noncommercial use, distribution, and reproduction in any medium, provided the original author(s) and source are credited.

\section{References}

1. Turner RC, Cull CA, Frighi V, Holman RR (1999) Glycemic control with diet, sulphonylurea, metformin, or insulin in patients with type 2 diabetes mellitus: progressive requirement for multiple therapies (UKPDS 49). UK Prospective Diabetes Study (UKPDS) Group. JAMA 281:2005-2012

2. Holst JJ (2002) Therapy of type 2 diabetes mellitus based on the actions of glucagon-like peptide-1. Diabetes Metab Res Rev 18:430-441

3. Holst JJ (2007) The physiology of glucagon-like peptide 1 . Physiol Rev 87:1409-1439

4. Nauck MA, Meier JJ, Creutzfeldt W (2003) Incretins and their analogues as new antidiabetic agents. Drug News Perspect $16: 413-422$

5. Knudsen LB, Nielsen PF, Huusfeldt PO et al (2000) Potent derivatives of glucagon-like peptide-1 with pharmacokinetic properties suitable for once daily administration. J Med Chem 43:1664-1669

6. Elbrønd B, Jakobsen G, Larsen S et al (2002) Pharmacokinetics, pharmacodynamics, safety and tolerability of a single dose of NN2211, a long-acting glucagon-like peptide 1 derivative, in healthy male patients. Diabetes Care 25:1398-1404

7. Agersø H, Jensen LB, Elbrønd B, Rolan P, Zdravkovic M (2002) The pharmacokinetics, pharmacodynamics, safety and tolerability of NN2211, a new long-acting GLP-1 derivative, in healthy men. Diabetologia 45:195-202

8. Degn KB, Juhl CB, Sturis J et al (2004) One week's treatment with the long-acting GLP-1 derivative, liraglutide (NN2211), markedly improves 24-h glycemia, alpha- and beta-cell function and reduces endogenous glucose release in patients with type 2 diabetes. Diabetes 53:1187-1194

9. Vilsbøll T, Zdravkovic M, Le-Thi T et al (2007) Liraglutide, a long-acting human glucagon-like peptide-1 analog, given as monotherapy significantly improves glycemic control and lowers body weight without risk of hypoglycemia in patients with type 2 diabetes. Diabetes Care 30:1608-1610

10. Heine RJ, Van Gaal L, Johns D, Mihm MJ, Widel MH, Brodows RG, for the GWAA Study Group (2005) Exenatide vs insulin glargine in patients with suboptimally controlled type 2 diabetes: a randomized trial. Ann Intern Med 143:559-569

11. Davies M, Storms F, Shutler S, Bianchi-Biscay M, Gomis R, for the ATLANTUS study group (2005) Improvement of glycemic control in participants with poorly controlled type 2 diabetes: comparison of two algorithms using insulin glargine. Diabetes Care 28:1282-1288

12. American Diabetes Association (2008) Standards of medical care in diabetes-2008. Diabetes Care 31(Suppl 1):S12-S54

13. AACE Diabetes Mellitus Clinical Practice Guidelines Task Force (2007) American Association of Clinical Endocrinologists medical guidelines for clinical practice for the management of diabetes mellitus. Endocr Pract 13(Suppl 1):1-66

14. Rosenstock J, Schwartz SL, Clark CM Jr, Park GD, Donley DW, Edwards MB (2001) Basal insulin therapy in type 2 diabetes: 28week comparison of insulin glargine (HOE 901) and NPH insulin. Diabetes Care 24:631-636 
15. Yki-Jarvinen H, Dressler A, Ziemen M (2000) Less nocturnal hypoglycemia and better post-dinner glucose control with bedtime insulin glargine compared with bedtime NPH insulin during insulin combination therapy in type 2 diabetes. HOE 901/3002 Study Group. Diabetes Care 23:1130-1136

16. Massi Benedetti M, Humburg E, Dressler A, Ziemen M (2003) A one-year, randomised, multicentre trial comparing insulin glargine with NPH insulin in combination with oral agents in patients with type 2 diabetes. Horm Metab Res 35:189-196

17. Fritsche A, Schweitzer MA, Haring HU (2003) Glimepiride combined with morning insulin glargine, bedtime neutral protamine Hagedorn insulin, or bedtime insulin glargine in patients with type 2 diabetes. A randomized, controlled trial. Ann Intern Med 138:952-959

18. Kennedy L, Herman WH, GOAL A1C Study Team (2005) Glycated hemoglobin assessment in clinical practice: comparison of the A1cNow point-of-care device with central laboratory testing (GOAL A1C Study). Diabetes Technol Ther 7:907-912

19. Janka HU, Plewe G, Riddle MC, Kliebe-Frisch C, Schweitzer MA, Yki-Jarvinen H (2005) Comparison of basal insulin added to oral agents vs twice-daily premixed insulin as initial insulin therapy for type 2 diabetes. Diabetes Care 28:254-259

20. Raskin P, Allen E, Hollander P et al (2005) Initiating insulin therapy in type 2 diabetes: a comparison of biphasic and basal insulin analogs. Diabetes Care 28:260-265

21. Riddle MC, Rosenstock J, Gerich J (2003) The treat-to-target trial: randomized addition of glargine or human NPH insulin to oral therapy of type 2 diabetic patients. Diabetes Care 26:3080-3086

22. Flint A, Kapitza C, Hindsberger C, Zdravkovic M (2008) The once-daily human GLP-1 analogue liraglutide improves both absolute and baseline corrected postprandial glucose levels. Diabetes 57(Suppl 1):A165
23. Courreges JP, Vilsboll T, Zdravkovic M et al (2006) Beneficial effects of once-daily liraglutide, a human glucagon-like peptide-1 analogue, on cardiovascular risk biomarkers in patients with type 2 diabetes. Diabet Med 25:1129-1131

24. Gutzwiller JP, Tschopp S, Bock A et al (2004) Glucagon-like peptide 1 induces natriuresis in healthy participants and in insulinresistant obese men. J Clin Endocrinol Metab 89:3055-3061

25. Horowitz M, Vilsbøll T, Zdravkovic M, Hammer M, Madsbad S (2008) Patient-reported rating of gastrointestinal adverse effects during treatment of type 2 diabetes with the once-daily human GLP-1 analogue, liraglutide. Diab Obes Metab 10:593-596

26. Bode B, Hale P, Hammer M, Testa MA, Garber A (2008) Patient reported outcomes in participants with type 2 diabetes treated with monotherapy regimens of once-daily human GLP1 analogue liraglutide or glimepiride. Diabetologia 51(1):S357 (Abstract 894)

27. Buse JB, Henry RR, Han J, Kim DD, Fineman MS, Baron AD, Exenatide-113 Clinical Study Group (2004) Effects of exenatide (exendin-4) on glycemic control over 30 weeks in sulfonylureatreated patients with type 2 diabetes. Diabetes Care 27:2628-2635

28. Kendall DM, Riddle MC, Rosenstock J et al (2005) Effects of exenatide (exendin-4) on glycemic control over 30 weeks in patients with type 2 diabetes treated with metformin and a sulfonylurea. Diabetes Care 28:1083-1091

29. Marre M, Shaw J, Brandle M et al (2009) Liraglutide, a once-daily human GLP-1 analogue, added to a sulphonylurea over 26 weeks produces greater improvements in glycaemic and weight control compared with adding rosiglitazone or placebo in participants with type 2 diabetes (LEAD-1 SU). Diabet Med 26:268-278

30. de Heer J, Holst JJ (2007) Sulfonylurea compounds uncouple the glucose dependence of the insulinotropic effect of glucagon-like peptide 1. Diabetes 56:438-443 